\title{
Das Mikroklima im Nistkasten und seine Auswirkungen auf die Nestlinge beim Star (Sturnus vulgaris)
}

\author{
Claudia Erbelding-Denk und Fritz Trillmich
}

\section{Einleitung}

Die Brutgröße der Vögel wird wesentlich durch das Nahrungsangebot zur Zeit der Jungenaufzucht bestimmt (LACK 1954, 1968). Bei Höhlenbrütern spielt jedoch auch die Größe der Bruthöhle eine Rolle (LÖHRL 1970, 1973, 1980; Moekd \& Dawson 1979; Gustafsson \& NiLsson 1985; Trillmich \& Hudde 1984): Meisen, Fliegenschnäpper und Stare (Sturnus vulgaris) ziehen große Kästen kleinen vor und legen in großen Kästen mehr Eier (s. jedoch KarLssON \& NiLsSON 1977). Welche Faktoren proximat und ultimat diese Präferenzen und die Abhängigkeit der Gelegegröße von der Kastengröße bestimmen, ist bisher unklar. Da große Starbruten in kleinen Kästen unter hoher Mortalität leiden (TRILlmich \& HudDE 1984), war zu vermuten, daß die Bedingungen im Nistkasten während der Aufzucht kritisch sind. Die Veränderung der Gelegegröße mit der Kastengröße könnte also einen maximalen Bruterfolg bei einer gegebenen Kastengröße sicherstellen.

Beim Bienenfresser (Merops apiaster) sinkt in den Bruttunneln bei ungünstigen Verhältnissen der $\mathrm{O}_{2}$-Gehalt der Luft von normalerweise $20,95 \%$ bis auf $15,8 \mathrm{Vol} \%$ und die $\mathrm{CO}_{2}$-Konzentration steigt von $0,03 \%$ auf 6,5 Vol\% (White et al. 1978). Auffällig war auch die hohe $\mathrm{NH}_{3}$-Anreicherung der Luft bis auf $718 \mathrm{ppm}$. Durch hohe $\mathrm{NH}_{3}$-Konzentration in der Atemluft kann die Infektionsanfälligkeit von Jungvögeln erhöht und gleichzeitig das Wachstum der Jungen verlangsamt werden (bei Hühnern; GRATZL \& KÖHIER 1968). So könnten mikroklimatische Bedingungen kleinere Bruten in kleinen Nistkästen begünstigen. Auch extreme Temperaturen sind - besonders bei hoher Luftfeuchtigkeit - eine Gefahr für das Überleben der Jungvögel (vAN BALEN 1984). Ab etwa $25^{\circ} \mathrm{C}$ müssen nämlich Starennestlinge, die älter als 9 Tage sind, Energie aufwenden, um genügend Wärme abzuführen (WesterTERP 1973). Die Jungen versuchen sich abzukühlen, indem sie stark Hecheln. Dieser Mechanismus versagt aber bei hoher Luftfeuchtigkeit im Kasten. Dadurch könnte bei hoher Temperatur im Kasten die letale Körpertemperatur der Jungvögel (bei Meisen ca. $45^{\circ} \mathrm{C}$, MerTENS 1977) schnell erreicht werden.

Wir haben versucht, die mikroklimatischen Bedingungen im Starenkasten genauer zu erfassen. Um abzuschätzen, welche Faktoren für das Überleben der Jungen kritisch werden könnten, maßen wir die Lufttemperatur $\left(\mathrm{T}_{\mathrm{a}}\right)$ und relative Luftfeuchtigkeit $\left(\mathrm{F}_{\mathrm{a}}\right)$ in der Umgebung des Kastens und im Kasten $\left(\mathrm{T}_{\mathrm{i}}, \mathrm{F}_{\mathrm{i}}\right)$, sowie den $\mathrm{O}_{2}$-, $\mathrm{CO}_{2}$ - und $\mathrm{NH}_{3}$-Gehalt der Luft in großen und kleinen Starenkästen. Die vorliegende Arbeit 
stellt keine abgeschlossene Analyse der Bedeutung mikroklimatischer Faktoren für die Jungenaufzucht bei Staren dar, sondern möchte in erster Linie die weitere Arbeit an diesem interessanten Problem stimulieren.

\section{Material und Methoden}

Die Kolonie bestand aus 30 Holznistkästen, die in $3 \mathrm{~m}$ Höhe an Stangen oder Bäumen entlang eines Altwassers der Ammer südlich des Ammersees bei Fischen (Kreis Weilheim/Schongau, Obb.) angebracht waren. 25 dieser Kästen hingen in einem nach SO offenen Halbrund um eine Wiese, die nach $\mathrm{N}, \mathrm{W}$ und $\mathrm{O}$ durch Gebüsch vor Wind geschützt war. Fünf weitere Kästen hingen in einem unmittelbar anschließenden lockeren Baumbestand. Die maximale Windgeschwindigkeit im Bereich der Kolonie betrug 1,3 m/s, lag normalerweise aber weit darunter, weil die Kolonie durch ein Halbrund von Bäumen nach Westen, Norden und Osten geschützt ist.

Am Anfang der Saison waren 30 große Holznistkästen vorhanden (361 cm² Bodenfläche, Gesamthöhe $28 \mathrm{~cm}$, Flugloch $48 \mathrm{~mm}$ Durchmesser in $17 \mathrm{~cm}$ Höhe über dem Kastenboden). Nach dem Schlüpfen der Bruten wurden dann 1985 zwischen dem 3. und 14. Lebenstag der Jungen 11 Bruten in kleine Nistkästen $\left(154 \mathrm{~cm}^{2}\right.$ Bodenfläche, sonst gleiche Maße) umgesetzt. 1986 wurden vier Bruten am 5. Lebenstag umgesetzt. Gleichzeitig wurden die Bruten durch Umsetzen gleichaltriger Jungvögel auf eine Größe von 4, 5 oder 6 Jungen eingestellt. Schlupfund Ausflugdaten der Jungen wurden durch regelmäßige Nistkastenkontrollen festgestellt. Der Tag des Schlüpfens galt als erster Lebenstag. Die Jungen wurden am 5., 10. und 15. Lebenstag gewogen. Alle angegebenen Uhrzeiten entsprechen der mitteleuropäischen Sommerzeit.

Die Messung der Parameter des Mikroklimas erfolgte auf zwei Weisen: (1) Longitudinal wurden die im folgenden genannten Meßgrößen 1985 mittags zwischen 11.00 und 14.00 Uhr über die gesamte Brutzeit gemessen, um den jahreszeitlichen Verlauf und den Streubereich der Werte zu erfassen. (2) An einigen Tagen der Brutzeiten 1985 und 1986 wurden die Parameter im Verlauf des Tages wiederholt gemessen, um den Tagesgang der Meßgrößen zu dokumentieren.

Temperaturmessungen: Am 27. 4. 1985 wurden Lufttemperatur und Luftfeuchtigkeit im leeren Kasten und vom 5. -7. 5. 1985 im Kasten mit ektothermen ( $\leqq 9$ Tagen alten) Jungen parallel alle 6 min gemessen (Datenspeichergerät DS 101, Firma MES-Pfau). Der Temperatursensor war an der Kastenrückwand in Kopfhöhe der Jungen befestigt, der Feuchtesensor $(60 \times 33 \times 140 \mathrm{~mm})$ mußte aus Platzgründen innen am Dach des Kastens angebracht werden. Ab 20. Mai bis zum 23. Juni wurde dies Datenspeichergerät - mit zwei Temperatursensoren bestückt - zur parallelen Messung der Lufttemperatur im Kasten $\left(T_{i}\right)$ und der Lufttemperatur außen $\left(T_{a}\right)$, im Schatten des Kastens, eingesetzt. Am 22. April (leere Kästen) und zwischen dem 18. Mai und dem 14. Juli 1985 (Kästen mit endothermen ( $\geqq 10$ Tage alten) Jungen, sowie bei den Messungen 1986 wurden $T_{i}$ und $T_{a}$ direkt mit einem $\mathrm{Ni}$-Cr-Ni Thermoelement in Verbindung mit einem digital anzeigenden Thermometer (2220-5 Ahlborn) gemessen.

Messung der relativen Luftfeuchtigkeit: Bei Kontrollmessungen am leeren Kasten am 22. 4. 1985 und Messungen am Kasten mit endothermen Jungen zwischen dem 29. Mai und dem 19. Juni wurde die Luftfeuchtigkeit in den Nistkästen $\left(\mathrm{F}_{\mathrm{i}}\right)$ mit einem Sekundenhygrometer ( 6400 Testoterm) in der Zeit zwischen 11.00 und 14.00 Uhr bestimmt. Die Messung erfolgte durch das Flugloch in Höhe der Köpfe der Jungen. Parallel maßen wir auch die relative Luftfeuchtigkeit außerhalb der Kästen $\left(F_{\mathrm{a}}\right)$. 
Sauerst offgehalt: Zur O2-Messung diente eine $\mathrm{O}_{2}$-Elektrolyt (Wissenschaftlich-Technische Werke, Weilheim), der vor jeder Messung auf Außenluft $\left(20,95 \mathrm{Vol} \% \mathrm{O}_{2}=100 \%\right)$ geeicht wurde. Da mit diesem Gerät die Bestimmung des $\mathrm{O}_{2}$-Gehaltes wegen der langsamen Gleichgewichtseinstellung des Elektrolyten mehrere Minuten dauerte, wurden insgesamt nur 47 Messungen zwischen dem 15. und dem 19. Mai 1985 vorgenommen, um die Kolonie nicht zu stark zu stören.

Kohlendioxid- $\left(\mathrm{CO}_{2}\right)$ und Ammoniak- $\left(\mathrm{NH}_{3}\right)$ Gehalt: Die Messungen wurden mit Prüfröhrchen der Firma Draeger $\left(0,1 \%\right.$ a und $0,5 \%$ a für $\mathrm{CO}_{2}$, bzw. $5 \%$ a und $0,5 \%$ a für $\mathrm{NH}_{3}$ ) vom 18. 5. bis 14. 7. 1985 durchgeführt. Zur Messung wird ein definiertes Volumen Kastenluft durch das Prüfröhrchen gezogen und das Ergebnis direkt an einer Meßskala am Prüfröhrchen abgelesen.

Nicht normalverteilte Daten wurden mit nicht-parametrischen Verfahren geprüft (SIEGEL 1957).

Danksagung: Wir danken B. Knauer und D. Schmide für die Zeichnungen und H. Biebach, E. GwinNer und W. WickLer für konstruktive Kritik des Manuskriptes. Unser herzlicher Dank gilt auch den Wissenschaftlichen Technischen Werken, Weilheim, die uns freundlicherweise ein Gerät zur Messung der Sauerstoffkonzentration im Nistkasten ausliehen.

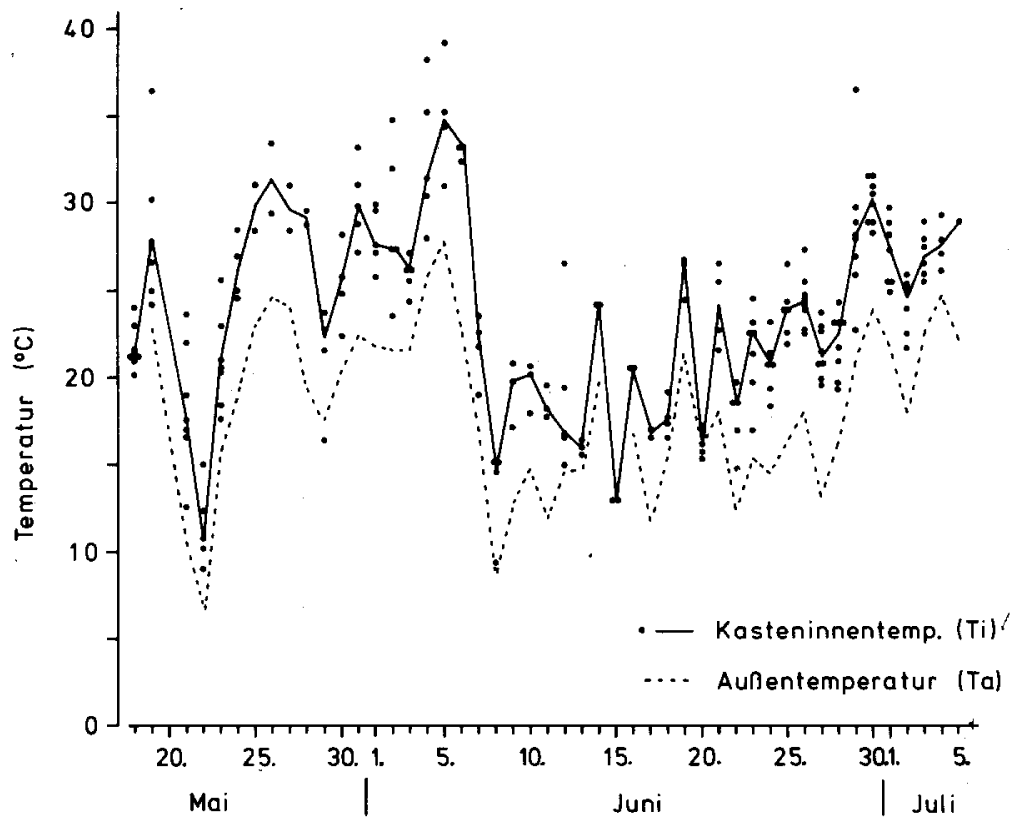

Abb. 1. Mittägliche Kasteninnentemperatur $\left(\mathrm{T}_{\mathrm{i}}\right)$ und Außentemperatur ( $\mathrm{T}_{\mathrm{a}}$, gestrichelt 18. 5. -15. 7. 1985. Punkte: Einzelmessungen von $\mathrm{T}_{\mathrm{i}}$ (in großen und kleinen Kästen); durchgezogene Linie: Median von $T_{i}$. - Air temperature at noon inside $\left(T_{i}\right)$ and outside $\left(T_{a}\right.$, hatched) the nest-box from May 18 to July 15, 1985. Values of $\mathrm{T}_{\mathrm{i}}$ (in small and large nest-boxes) are dots, continuous line is the median of $\mathrm{T}_{\mathrm{i}}$. 
Temperatur

\section{Ergebnisse}

1985 stieg die Mittags-Lufttemperatur $\left(\mathrm{T}_{\mathrm{a}}\right)$ von niedrigen Werten Ende April auf ein Maximum von $28^{\circ} \mathrm{C}$ Anfang Juni und fiel dann abrupt auf $8^{\circ} \mathrm{C}$ ab. Erst Mitte Juni lagen die Mittagstemperaturen der Luft wieder bei $15^{\circ} \mathrm{C}$ (Abb. 1).

Die Innentemperatur $\left(T_{\mathrm{i}}\right)$ in Kästen mit endothermen Jungen ( $>10$ Tage alt) korrelierte mit $T_{a}(r=0,87, P<0,001, N=47)$. Kontrollmessungen zeigten den Einfluß der Kästen auf $\mathrm{T}_{\mathrm{i}}$. Durch Absorption von Strahlung und die Isolationswirkung der Kästen lag $T_{i}$ auch in Kästen, die nur Nester enthielten (mit oder ohne Eier), Ende April um $1-3^{\circ} \mathrm{C}$ über $\mathrm{T}_{2}$.

In Kästen mit endothermen Jungen lag $\mathrm{T}_{\mathrm{i}}$ in den Mittagsstunden um $5,8 \pm 2,5^{\circ} \mathrm{C}$ $(N=227)$ über $T_{a}$ (Abb. 1). $T_{i}$ zeigte einen deutlichen Tagesgang (Abb. 2 und $\left.4 a\right)$ und ein breites Maximum am Nachmittag (Abb. 2). Die Differenz zwischen $T_{i}$ und $T_{a}$ wurde durch die Anzahl der Jungen in einem Kasten schwach beeinflußt $\left(r_{s}=\right.$ $0,24, P<0,001, N=227$, was auch am Temperaturverlauf in einzelnen Kästen nach Zusetzen oder Tod von Jungen zu beobachten war. Bei den höchsten gemessenen $\mathrm{T}_{\mathrm{i}}$ (max. $39,1^{\circ} \mathrm{C}$ ) trat keine akute Mortalität der Jungvögel auf.

Bei gleicher Anzahl (5) gleichaltriger (16-17tägiger) Junger war die $T_{i}$ im großen Kasten niedriger als im kleinen (Abb. 2; Wircoxon Test, $\mathrm{P}<0,01 ; \mathrm{N}=16$; Daten von 1986).

Während der Aufzucht der Jungen wurden das Nestmaterial und die Kastenwände durchnäßt. Dementsprechend erniedrigte sich $T_{i}$ nach dem Ausfliegen der Jungen durch Wasserverdunstung und lag dann um bis zu $3{ }^{\circ} \mathrm{C}$ niedriger als $\mathrm{T}_{\mathrm{a}}$ (gemessen am 9. 6., 12. 6. und 23. 6. 1985).

\section{Relative Luftfeuchtigkeit}

Am 22. und 27. April wurden Luftfeuchtigkeit $\left(F_{i}\right)$ und $T_{i}$ in Kästen ohne Junge gemesseri. Bei einer Mittagss $\mathrm{T}_{i}$ am 22. 4. zwischen $21,7^{\circ} \mathrm{C}$ und $28,4^{\circ} \mathrm{C}$ lag die $\mathrm{F}_{\mathrm{i}}$ im

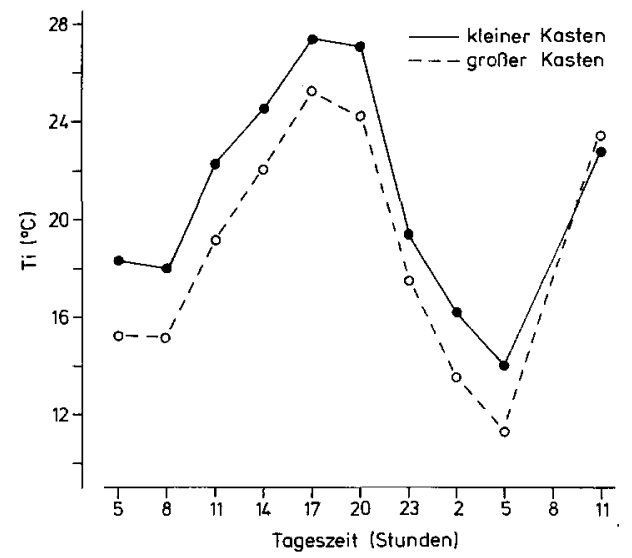

Abb. 2. Tagesgang der Lufttemperatur $T_{i}$ im Kasten mit fünf 16tägigen Jungen (22./23. 5. 1986). - Air temperature $\left(\mathrm{T}_{\mathrm{i}}\right)$ in nest-boxes with 5,16-days old young on May 22/23 1986. 


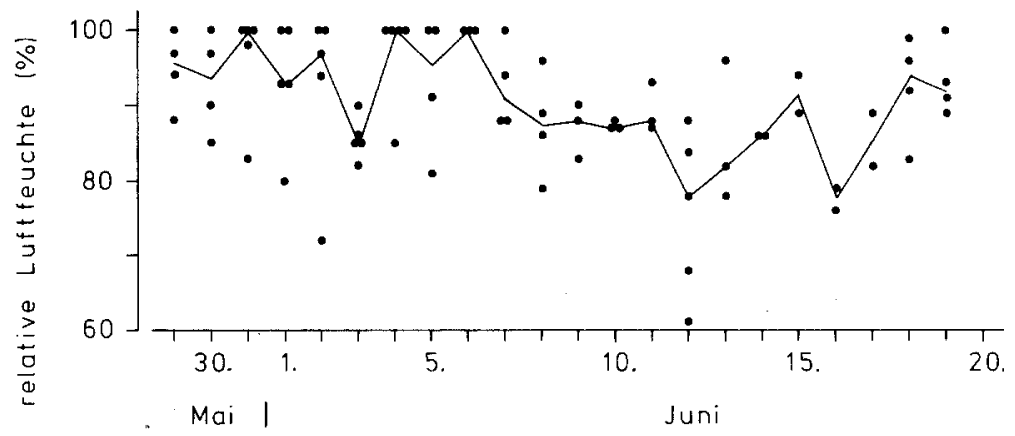

Abb. 3. Relative Luftfeuchtigkeit $\left(F_{i}\right)$ 29. 5. -19. 6. 1985. Punkte: Einzelmessungen. Linie: Tagesmedian. - Relative humidity $\left(F_{i}\right)$ in nest-boxes between May 29 and June 19, 1985. Dots: individual measurements; line: daily median.

Kasten zwischen $49 \%$ und $59 \%$. Ebenso stieg die $\mathrm{F}_{\mathrm{i}}$ im Tagesverlauf am 27. 4. bei $\mathrm{T}_{\text {i }}$ 's zwischen $2{ }^{\circ} \mathrm{C}$ und $13^{\circ} \mathrm{C}$ nie über $75 \%$.

In Nistkästen mit Jungen lag $F_{i}$ drastisch höher. Das galt für Kästen mit ektothermen Jungen im Alter von 6 Tagen, aber insbesondere für Kästen mit älteren, bereits endothermen Jungen (Abb. 3 für Mittagswerte von $F_{i}$ im Mai und Juni). In der Außenluft ist aus physikalischen Gründen eine negative Korrelation zwischen $F_{a}$ und $T_{\mathrm{a}}$ zu erwarten. In Kästen mit Jungen im Alter von $\geqq 10$ Tagen korrelierten $T_{\mathrm{i}}$ und $F_{i}$ dagegen positiv $\left(r_{s}=0,63 ; P<0,001, N=81\right) . F_{i}$ lag hier im Mittel bei $90 \pm 8,4 \%$ (Bereich $61-100 \%$; Abb. 3). Ab einer $\mathrm{T}_{\mathrm{i}}$ von $30^{\circ} \mathrm{C}$ lag $\mathrm{F}_{\mathrm{i}}$ immer bei $100 \%$.

Weder die Luftfeuchtigkeit außerhalb des Kastens $\left(F_{2}\right)$, noch die Jungenzahl im Kasten korrelieren signifikant mit $\mathrm{F}_{\mathrm{i}}$ (für $\mathrm{F}_{\mathrm{a}}: \mathrm{r}=0,132, \mathrm{P}>0,05, \mathrm{~N}=81$; für Jungenzahl: $r=0,13, P>0,05, N=66$ ). Da diese Werte mittags gemessen wurden, war $F_{i}$ meist schon in der Nähe von $100 \%$. In großen und kleinen Kästen mit Jungtieren war $\mathrm{F}_{\mathrm{i}}$ nicht signifikant verschieden (MANN-WHITNEY $U$ Test, $\mathrm{P}=0,9$ ).

\section{Hygienische Situation im Kasten}

Während der Aufzucht der Jungen änderte sich die hygienische Situation in den Kästen stark. Schon die durch Verdunstungskälte erniedrigte $T_{1}$ nach dem Ausfliegen der Jungen weist auf hohe Feuchtigkeit in den Kästen hin. Besonders schlechte Bedingungen entstanden in kleinen Kästen. Wenige Tage nach dem Umsetzen der Jungen aus großen in kleine Kästen löste sich im Jahre 1985 das Nest auf. Die Jungen saßen in einer bis zu 1,5 cm dicken Schlammschicht, von der sie selbst besonders an Beinen und Bauch überkrustet waren. Dieser Effekt war 1986 sehr viel schwächer.

\section{Sauerstoffgehalt}

Die Mehrzahl der O2-Messungen (26 von 47) wurden am 19. Mai über den ganzen Tag verteilt an 7 Nistkästen parallel vorgenommen (Abb. 4d). Oz-Messungen wurden 

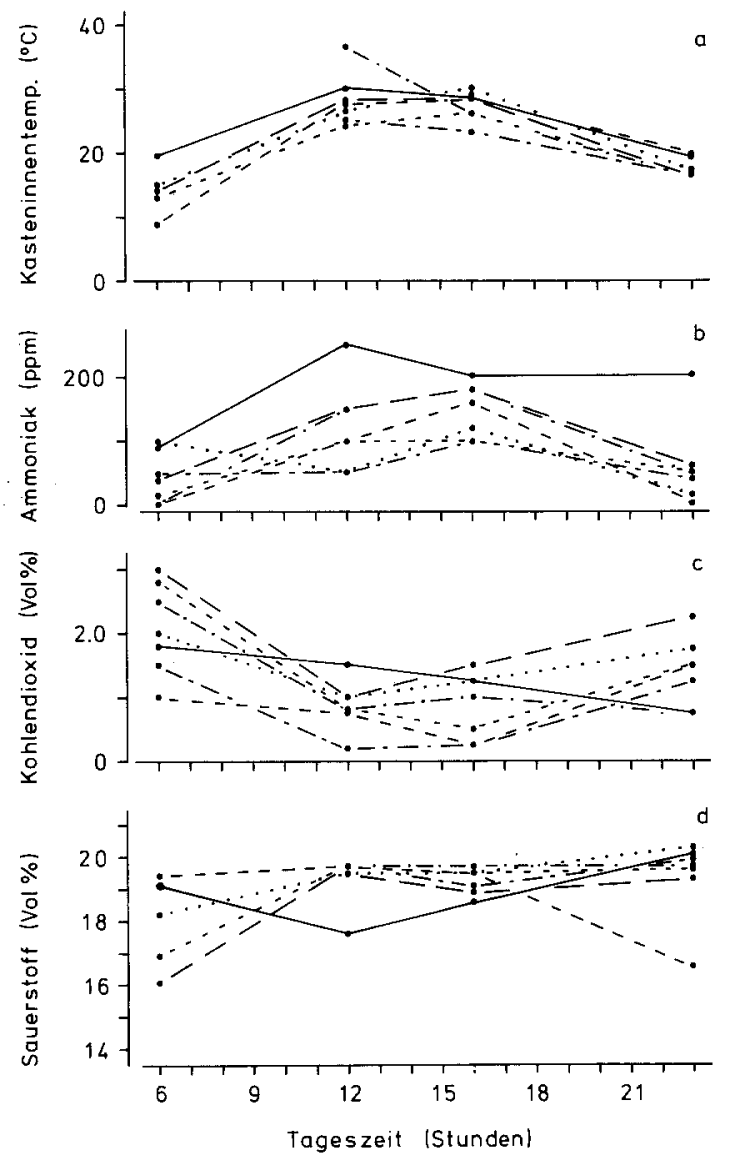

Abb. 4. Messungen am 18. 5. 1985 von a) $\mathrm{T}_{\mathrm{i}}$, b) $\mathrm{NH}_{3}$, c) $\mathrm{CO}_{2}$, d) $\mathrm{O}_{2}$ in 7 Brutkästen. Meßzeiten: 6.00, $12.00,16.00,23.00 \mathrm{Uhr}$. Identische Werte und sich überdeckende $\mathrm{Li}$ nien sind einfach eingezeichnet. Unterschiedliche Linien kennzeichnen verschiedene Kästen. Measurements of a) $\mathrm{T}_{\mathrm{i}}, \mathrm{b}$ ) $\mathrm{NH}_{3}, \mathrm{c}$ ) $\mathrm{CO}_{2}$, d) $\mathrm{O}_{2}$ in 7 nest-boxes on 18 May 1985 at 6.00, 12.00, 16.00 and $23.00 \mathrm{hrs}$. Identical values and overlapping lines are drawn singly. Different lines for different nest-boxes.

an Kästen vorgenommen, in denen die Jungenzahl zwischen 3 und 6 Jungen im Alter von 5 bis 19 Tagen bei $\mathrm{T}_{i}$-Werten zwischen $8^{\circ} \mathrm{C}$ und $36^{\circ} \mathrm{C}$ variierten. Im Mittel lag der $\mathrm{O}_{2}$-Gehalt bei $19,3 \pm 1,0 \mathrm{Vol} \%(\mathrm{~N}=47)$. Morgens um 6.00 war der $\mathrm{O}_{2}$-Gehalt der Luft in den Kästen am geringsten $(18,0 \pm 1,4 \mathrm{Vol} \%, \mathrm{~N}=5)$ (Abb. 4d). Von 12.00-23.00 Uhr blieb er fast konstant bei $19 \mathrm{Vol} \%(\mathrm{~N}=21)$. Der niedrigste $\mathrm{O}_{2}$-Gehalt der Kastenluft betrug um 6.00 Uhr in einem großen Kasten mit sechs 15tägigen Jungen 16,1 Vol\%. Der Höchstwert entsprach dem $\mathrm{O}_{2}$-Gehalt der Außenluft $(20,95$ Vol.\%).

Der $\mathrm{O}_{2}$-Gehalt im Kasten zeigte keine Abhängigkeit vom Jungenalter $(\mathrm{r}=-0,09$, $\mathrm{P}=0,66)$, der $\mathrm{T}_{\mathrm{i}}(\mathrm{r}=0,09, \mathrm{P}=0,14)$, der Jungenzahl (MANN-WHITNEY U-Test für 4 gegen 6 Junge, $z=0,41$, n. s.) und der Kastengröße (MANN-WhitNey UTest, $z=0,1$, n. s.). 


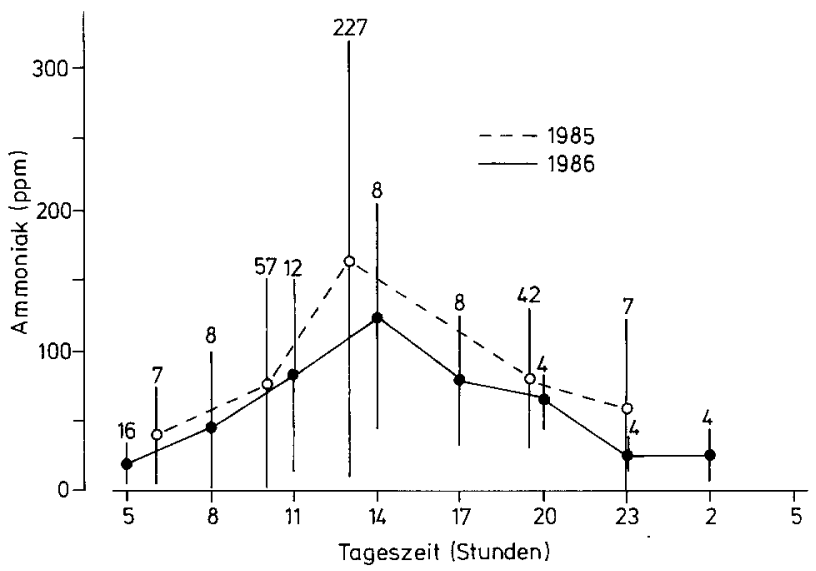

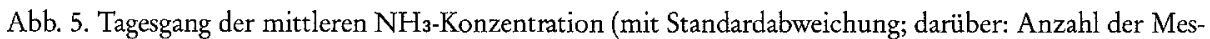
sungen) In Kästen 1985 (18. 5. -5. 7.) und 1986 (15. - 23. 5.). - Daily course of mean $\mathrm{NH}_{3}$ concentration (with standard deviation; above: number of measurements) in nest-boxes in 1985 (18 May to 5 July) and 1986 (15-23 of May).

\section{Kohlendioxid}

Der CO2-Gehalt der Kastenluft lag zwischen 8.00 und 20.00 Uhr bei $0,9 \pm 0,35$ Vol\% ( $\mathrm{N}=42$; Daten von 1986). Messungen um 6.00 und 23.00 Uhr ergaben höhere Werte von $1.7 \pm 0,7$ und $1,3 \pm 0,7$ Vol\% (jeweils $N=7 ;$ Abb. 4 c). Der Höchstwert von 3,0 Vol\% wurde am 19. Mai 1985 um 6.00 in einem großen Kasten mit sechs 15tägigen Jungen gemessen. Im Tagesverlauf nimmt die $\mathrm{CO}_{2}$-Konzentration also ab, wenn die $\mathrm{O}_{2}$-Konzentration zunimmt. Dieser Zusammenhang weist darauf hin, daß der $\mathrm{CO}_{2-}$ und $\mathrm{O}_{2}$-Gehalt im Kasten gemeinsam von der Ventilation des Kastens bestimmt werden, die tagsüber durch die Nistkastenbesuche der Eltern und die Bewegung der Jungen im Kasten höher ist.

Im Verlauf der Saison war der $\mathrm{CO}_{2}$-Gehalt mit der $\mathrm{T}_{\mathrm{i}}$ schwach positiv $\left(\mathrm{r}_{\mathrm{s}}=0,17\right.$, $\mathrm{P}<0,05, \mathrm{~N}=228)$ und mit der $\mathrm{F}_{\mathrm{i}}$ hochsignifikant positiv $\left(\mathrm{r}_{\mathrm{s}}=0,45 ; \mathrm{P}<0,001, \mathrm{~N}\right.$ $=228$ ) korreliert. Die Korrelation zwischen $\mathrm{O}_{2}$ - und $\mathrm{CO}_{2}$-Gehalt der Luft im Kasten war negativ $\left(r_{s}=-0,59, \mathrm{P}<0,001, N=43\right)$.

Unterschiede im $\mathrm{CO}_{2}$-Gehalt in Abhängigkeit von der Brutgröße und der Kastengröße ließen sich nicht sichern (Daten von 1986: WILCOxON-Test für 5 vs. 4 Junge bei gegebener Kastengröße und für kleinen gegen großen Kasten bei gegebener Zahl gleichaltriger Junger, immer n. s.).

\section{Ammoniak}

Der $\mathrm{NH}_{3}$-Gehalt im Nistkasten zeigte ebenfalls eine deutliche Tagesperiodik. Um 5.00-6.00 Uhr wurden die geringsten Werte gemessen; nach einem Anstieg zwischen 9.00 und 11.00 Uhr wurde das Maximum zwischen 12.00 und 14.00 Uhr erreicht. 


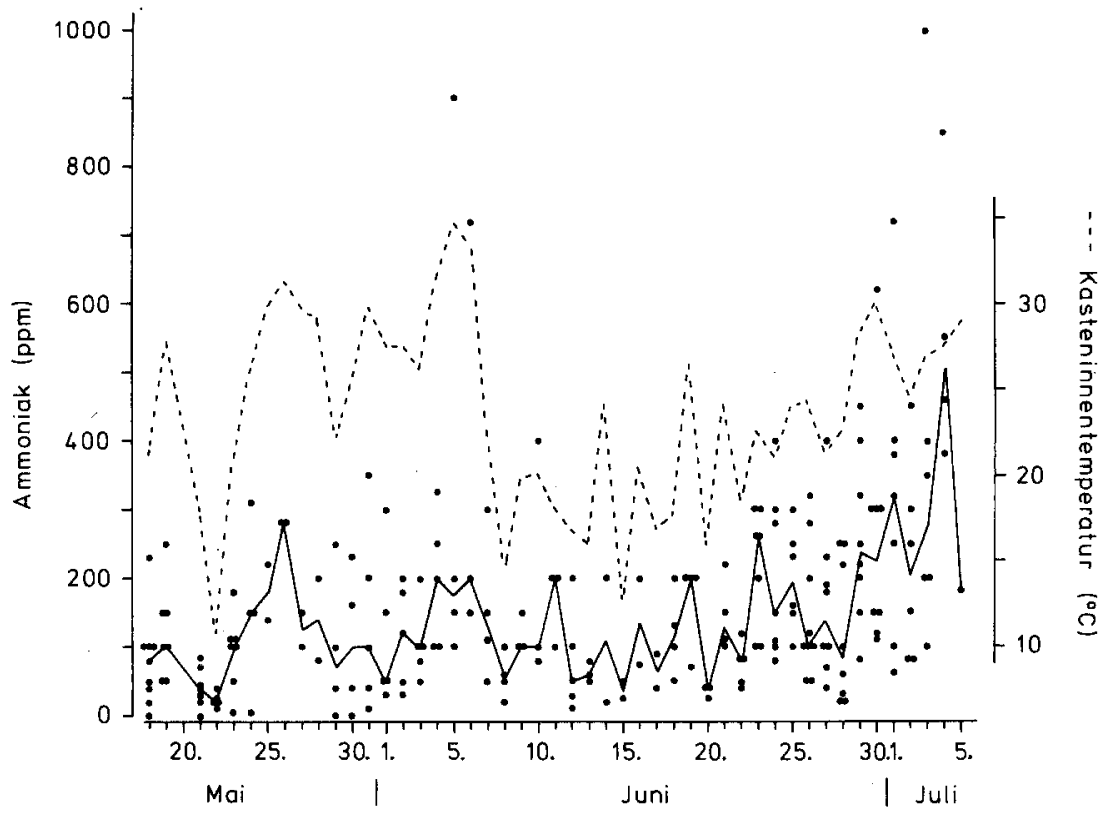

Abb. 6. Saisonaler Verlauf des Median des mittags in Nistkästen gemessenen $\mathrm{NH}_{3}-$ Gehaites der Luft (durchgezogene Linie) 18. 5. - 5. 7. 1985. Punkte: Einzelmessungen; gestrichelte Linie: Median der KastenInnentemperatur $\left(\mathrm{T}_{\mathrm{i}}\right)$. - Seasonal changes of median noon $\mathrm{NH}_{3}$ concentrations in nest-box air (line) from 18 May to 5 July 1985. Dots: individual measurements. Hatched line: median of simultaneously measured air temperature inside nest-box $\left(\mathrm{T}_{\mathrm{i}}\right)$.

Danach fiel die $\mathrm{NH}_{3}-$ Konzentration langsam wieder ab (Abb. 5). Der Tagesgang war bei Erstbruten 1986 gleichartig, verlief aber auf niedrigerem Niveau (Abb. 5). Maximal wurden 1986 nur 270 ppm NH3 gemessen, während das absolute Maximum am 3. Juli 19851000 ppm NH3 betrug (Abb. 6).

Korreliert man die Mittagswerte von $\mathrm{T}_{\mathrm{i}}$ und $\mathrm{F}_{\mathrm{i}}$ mit der $\mathrm{NH}_{3}$-Konzentration der Kastenluft über die Saison, erhält man positive Zusammenhänge $\left(T_{i}: r_{s}=0,38, P\right.$ $\left.<0,001, \mathrm{~N}=225 ; \mathrm{F}_{\mathrm{i}}: \mathrm{r}_{\mathrm{s}}=0,48, \mathrm{P}<0,001, \mathrm{~N}=81\right)$.

Bei Messungen in Kästen, die nach Jungenzahl, Jungenalter und Kastenstandort miteinander vergleichbar waren, lag der $\mathrm{NH}_{3}$-Gehalt der Luft in kleinen Kästen signifikant höher als in großen (Abb. 7; WircoXON Tests kleiner vs. großer Kasten; 1985: 4 Junge, 2. Brut: $z=2,2, P<0,03, N=13$. 1986: 4 Junge, 1 . Brut: $z=3,1, P<0,01$, $\mathrm{N}=13.5$ Junge, 1 . Brut: $\mathrm{z}=2,7, \mathrm{P}<0,01, \mathrm{~N}=16$ ).

Die mittlere Jungenmasse am 15. Lebenstag korrelierte negativ mit steigender, über 10 Tage gemittelter $\mathrm{NH}_{3}-\mathrm{Belastung}$ der Jungen im Kasten (Abb. 8; $\mathrm{r}=-0,44, \mathrm{P}=$ $0,004, \mathrm{~N}=24)$. 


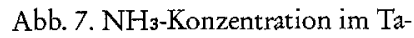
gesverlauf (22./23. 5. 1986; fünf 16tägige Junge). - $\mathrm{NH}_{3}$ concentration in small versus large nestboxes. (22/23 May 1986 in nestboxes with 5 16-day old nestlings).

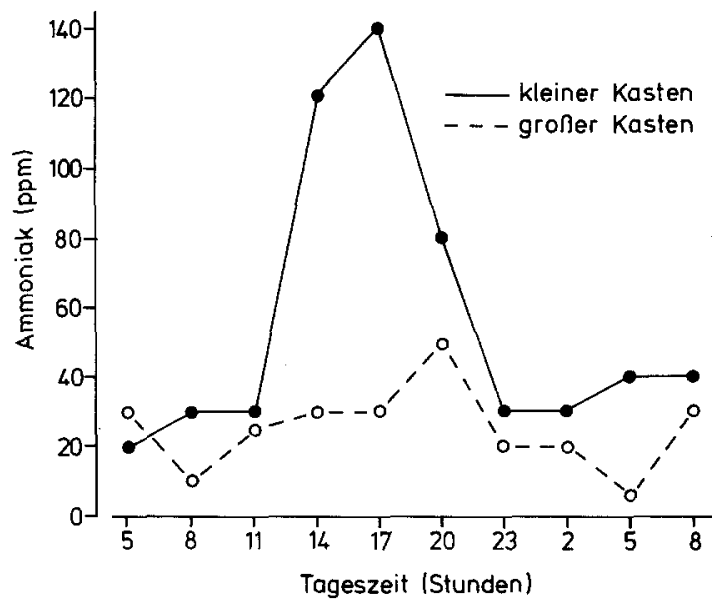

Abb. 8. Mittlere Jungenmasse am 15. Lebenstag und über 10 Tage gemittelte mittägliche $\mathrm{NH}_{3}$-Belastung. - Relation between mean nestling mass on day 15 and noon $\mathrm{NH}_{3}$ concentration in the nest-box

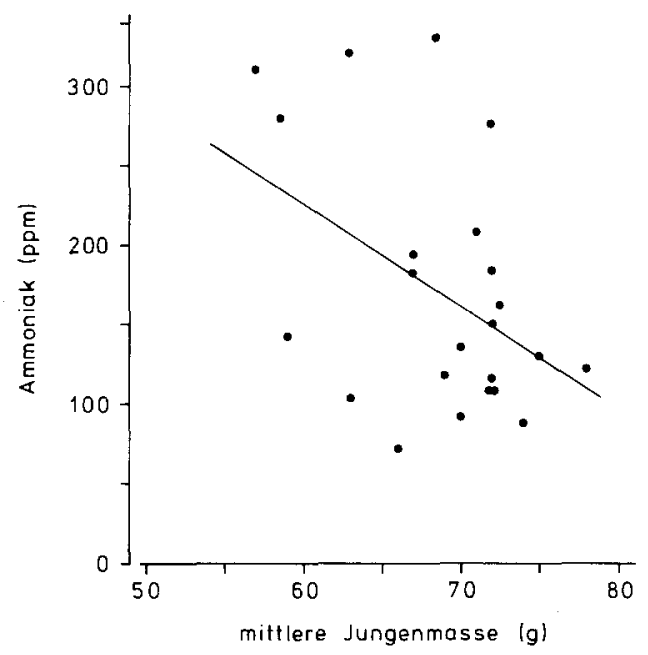
averaged over the previous 10 days.

\section{Diskussion}

\section{Tagesverlauf des Mikroklimas}

Durch steigende Temperatur verschlechterte sich in den Nistkästen im Laufe des Tages das Mikroklima. Einige Faktoren, die schon für sich allein eine Belastung für die Starenjungen bedeuten, verstärken sich dabei gegenseitig. Junge, die im Nistkasten eng zusammensitzen, können bei hohen Umgebungstemperaturen Wärme nur noch durch Verdunsten von Wasser abgeben. Wasser wird über die Lungen verdunstet, $d$. h. die Wasserabgabe wird durch Hecheln gefördert. Hohe Wasserverdunstung hat negative Effekte: Erstens atmen die Jungen viel $\mathrm{CO}_{2}$ ab, was potentiell zu einer Alkalose führen kann. Zweitens steigt dabei die Feuchte der Kastenluft bis zur Sättigung, was eine Abkühlung durch Wasserverdunstung immer mehr behindert. Außerdem 
bewirkt die Kombination hoher Temperatur mit hoher Luftfeuchtigkeit einen Anstieg der $\mathrm{NH}_{3}$-Konzentration. Das liegt daran, daß $\mathrm{NH}_{3}$ im Kasten wohl nur in geringem Maße von den Jungvögeln direkt ausgeschieden wird, sondern vielmehr zum größten Teil durch bakteriellen Abbau von Harnsäure und Harnstoff erzeugt wird. Das Wachstum von Bakterien wird durch hohe Temperatur und Luftfeuchtigkeit stark gefördert. Dieser Zusammenhang könnte die Korrelationen zwischen $\mathrm{F}_{\mathrm{i}}, \mathrm{T}_{\mathrm{i}}$ und $\mathrm{NH}_{3}-\mathrm{Gehalt}$ der Kastenluft erklären.

Atmen die Jungen mit der Umgebungsluft viel $\mathrm{NH}_{3}$ in hoher Konzentration ein, dürfte seine Wirkung auf die Physiologie der Körperflüssigkeit und auf das Epithel des respiratorischen Gewebes besonders gravierend sein. Hohe Temperatur scheint demnach der auslösende Faktor für die Verschlechterung des Mikroklimas im Nistkasten zu sein. Sie führt in kleinen Kästen anscheinend zu einem stärkeren Bakterienwachstum und somit zu höherem $\mathrm{NH}_{3}$-Gehalt der Luft und einer verschlechterten hygienischen Situation (Abb. 2, 7).

Diese Zusammenhänge könnten ultimat auch die Reduktion der Brutgröße in kleinen Kästen verursachen, da die Temperaturdifferenz $\left(T_{i}-T_{a}\right)$ und auch die absolute Temperatur in den Kästen mit der Jungenzahl ansteigt. Wenn wir im Laufe unserer Messungen auch keine akute Mortalität von Starenjungen nach Höchstwerten der Temperatur oder des $\mathrm{NH}_{3}$-Gehaltes der Luft im Kasten beobachteten, so ist die erhöhte Mortalität großer Bruten in kleinen Kästen vermutlich durch chronische Effekte der beschriebenen Art mitverursacht. Es wäre wünschenswert, diese Hypothese durch kontrollierte Begasung von Jungvögeln mit NHз-haltiger Luft zu testen.

\section{Saisonale Effekte}

Zunehmende Temperatur allein reichte beim Königstyrannen (Tyrannus tyrannus) bereits aus, das Wachstum von Nestjungen zu verlangsamen (MURPHY 1985). Trotz starker Schwankungen stiegen im Laufe der Brutzeit die Innentemperaturen der Nistkästen beim Star allmählich weiter an, wodurch wiederum $\mathrm{CO}_{2}$-Konzentration und Feuchte zunahmen (Abb. 1). Die im Laufe der Brutsaison voranschreitende bakterielle Verseuchung des Nistmaterials verursacht meist wohl höhere $\mathrm{NH}_{3}$-Konzentrationen bei Zweitbruten (Abb. 6). Die Belastung von Starenjungen durch das Mikroklima steigt daher im Laufe der Brutsaison. Zweitbruten leiden also möglicherweise unter schlechteren Aufzuchtbedingungen im Kasten. Das dürfte bei Naturhöhlen noch gravierender sein als bei den von uns gebotenen Nistkästen, da wir die Nester nach dem Ausfliegen der ersten Brut vollständig ausräumten und damit die Ansammlung fauligen Nistmaterials verhinderten. Stare selber räumen aber kaum Nistmaterial aus, sondern bauen ein dünnes frisches Nest auf das alte, was zu einer schnellen Durchseuchung des neuen Nestes der Zweitbrut führen dürfte. Dies könnte mit verantwortlich dafür sein, daß Junge in Zweitbruten langsamer wuchsen als in Erstbruten. Eine Verringerung der Jungenzahl in Zweitbruten könnte also unter vollständig natürlichen Bedingungen auch eine Anpassung an die schlechteren Bedingungen in der Nisthöhle darstellen und nicht nur auf verringerte Nahrungsverfügbarkeit für die Zweitbrut zurückzuführen sein. 


\section{Zusammenfassung}

Die Nistkastengröße beeinflußt nach früheren Untersuchungen die Überlebenschancen von Starenbruten (TRulmich \& HudDe 1984). Wir prüften, ob Unterschiede im Mikroklima in großen (361 cm $\mathrm{cm}^{2}$ Grundfläche) und kleinen Starennistkästen (154 $\mathrm{cm}^{2}$ Grundfläche) dafür ver-

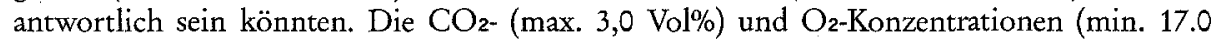
$\mathrm{Vol} \%$ ) in beiderlei Kästen waren nicht signifikant voneinander verschieden. Bei gleicher Brutgröße lagen die Innentemperaturen in kleinen Kästen höher als in großen. Maximal wurden $39,1^{\circ} \mathrm{C}$ in den Kästen gemessen. Die relative Luftfeuchtigkeit in Nistkästen mit Jungen lag im Mittel bei $90 \%$ und erreichte häufig $100 \%$. Ammoniak (NH3) trat in der Kastenluft in Konzentrationen bis zu 1000 ppm auf. Der $\mathrm{NH}_{3}-$ Gehalt der Luft war bei gleicher Brutgröße in kleinen Kästen signifikant höher als in großen. Die mittlere Jungenmasse der Bruten korrelierte negativ mit der mittleren $\mathrm{NH}_{3}$-Konzentration in der Kastenluft. Vermutlich tragen hohe Kastentemperatur in Verbindung mit hoher relativer Luftfeuchtigkeit und erhöhter $\mathrm{NH}_{3}-$ Konzentration zu der beobachteten höheren Jungenmortalität in kleinen Kästen bei. Außerdem waren kleine Kästen oft sehr viel stärker verschmutzt als große, was zu einer höheren Infektionsanfälligkeit der Jungvögel in kleinen Kästen führen könnte. Auf Grund saisonaler Effekte ist anzunehmen, daß Zweitbruten im allgemeinen stärker unter mikroklimatischen Belastungen leiden als Erstbruten.

\section{Summary}

Nestbox climate and its effects on Starling (Sturnus vulgaris) nestlings.

Nest-box size influences the survival probability of nestling starlings (TRILMICH \& HUDDE 1984). We investigated whether microclimatic differences between large nest-boxes $\left(361 \mathrm{~cm}^{2}\right.$ bottom area) and small ones $\left(154 \mathrm{~cm}^{2}\right)$ might be responsible for this difference. $\mathrm{CO}_{2}$ (max. $3.0 \mathrm{vol} \%$ ) and $\mathrm{O}_{2}$-concentrations ( $\mathrm{min} .17 .0 \mathrm{vol} \%$ ) were not significantly different between the two types of nest-box. At the same brood size, small nest-boxes experienced higher internal air temperatures than large ones. Maximal air temperature in a nest-box was $39^{\circ} \mathrm{C}$. Relative humidity in nest-boxes with chicks was $90 \%$ and frequently reached $100 \%$. At maximum, Ammonia- $\left(\mathrm{NH}_{3}\right)$ concentration in the nest-box air built up to $1000 \mathrm{ppm}$. At the same brood size, $\mathrm{NH}_{3}$-concentration in the nest-box air was higher in smaller nest-boxes than in larger ones. High internal air temperatures combined with near $100 \%$ relative humidity and increased $\mathrm{NH}_{3}$-concentrations presumably contribute to the increased chick mortality observed in small nestboxes. In addition, small nest-boxes were more likely to turn exceedingly dirty thus increasing the danger of chick infections. Due to seasonal increases in temperature and ammonia combined with a build-up of dirt in used nest-boxes it is to be expected that second broods suffer more than first broods from adverse micro-climatic conditions inside nest-boxes.

\section{Literatur}

Gratzi, E., \& H. Köhler (1968): Spezielle Pathologie und Therapie der Geflügelkrankheiten. Stuttgart. - Gustafsson, L., \& S. G. Nilsson (1985): Clutch size and breeding success of Pied and Collared flycatchers Ficedula spp. in nest-boxes of different sizes. Ibis 127: 380-385. • KarLSSON, J., \& S. G. NiLSSON (1977): The influence of nest-box area on clutch size in some hole-nesting passerines. Ibis 119: 207-211. - LACK, D. (1954): The natural regulation of animal numbers. Oxford. • Ders. (1968): Ecological adaptions for breeding in birds. London. • LÖHRL, H. (1970): Unterschiedliche Bruthöhlenansprïche von Meisenarten und Kleiber als Beitrag zum Nischenproblem. Verh. Dtsch. Zool. Ges. 64: 314-317. • Ders. (1973): Einfluß 
der Brutraumfläche auf die Gelegegröße der Kohlmeise (Parus major). J. Orn. 114: 339-347. - Ders. (1980): Weitere Versuche zur Frage „Brutraum und Gelegegröße” bei der Kohlmeise, Parus major. J. Orn. 121: 403-405. • MERTENS, J. A. (1977): Thermal conditions for successful breeding in Great tits (Parus major L.). Oecologia 28: 1-29. Moefd, A., \& D. G. Dawson (1987): Breeding of Starlings (Sturnus vulgaris) in nest-boxes of various types. New Zeal. J. Zool. 6: 613-618. • MuRPHY, M. T. (1985): Nestling eastern Kingbird growth: effects of initial size and ambient temperature. Ecology 66: 162-170. - SIEGEL, S. (1957): Nonparametric statistics. N Y. • Trillmich, F., \& H. Hudde (1984): Der Brutraum beeinflußt Gelegegröße und Fortpflanzungserfolg beim Star (Sturnus vulgaris). J. Orn. 125: 75-79. • vaN BaLEN, J. H. (1984): The relationship between nest-box size, occupation and breeding parameters of the great tit Parus major and some other hole-nesting species. Ardea 72:163-175. - WESTERTERP, K. (1973): the energy budget of the nestling starling Sturnus vulgaris, a field study. Ardea 61: 137-158. - WhtTe, F. N., G. A. Bartholomew, \& J. Kinney (1978): Physiological and ecological correlates of tunnel nesting in the European bee-eater, Merops apiaster. Physiol. Zool. 51: $140-154$.

Anschrift der Verfasser: Max-Planck-Institut für Verhaltensphysiologie, Abt. Wickler, D-8131 Seewiesen. 